\title{
Current Diagnostic Criteria for Neurocysticercosis
}

\author{
Carolina Guzman (D') \\ Hector H Garcia ${ }^{1,2}$ \\ for The Cysticercosis Working \\ Group in Peru \\ 'Center for Global Health, Universidad \\ Peruana Cayetano Heredia, Lima, Perú; \\ ${ }^{2}$ Cysticercosis Unit, Instituto Nacional de \\ Ciencias Neurológicas, Lima, Perú
}

Correspondence: Hector H Garcia Email hgarcia@jhsph.edu

\begin{abstract}
Neurocysticercosis (NCC) causes significant neurological morbidity around the world, and is the most common preventable factor for epilepsy in adults. It is endemic in most developing countries, and also diagnosed with some frequency in industrialized countries because of travel and migration. The clinical manifestations of NCC are extremely variable and may include almost any neurological symptom, depending on the number of lesions, location, size and evolutive stage of the infecting parasitic larvae and the immune response of the host. Thus, the diagnosis of NCC relies mostly on neuroimaging and immunological tests. Despite being a disease with a known etiology, the lack of specificity of clinical manifestations and auxiliary examinations makes its diagnosis difficult. In an attempt for developing a standard diagnosis approach, a chart of diagnostic criteria for NCC was initially published in 1996, and revised in 2001 and 2017. This chart of diagnostic criteria systematized the diagnosis of NCC and became widely used worldwide. This manuscript describes the structure of the chart, the principles behind the changes for each revision, as well as the context of its use and potential for improvement.
\end{abstract}

Keywords: cysticercosis, neurocysticercosis, Taenia solium, diagnosis, epilepsy, epidemiology

\section{Introduction}

Considered a major public health problem in most of the world, cysticercosis (infection with the larval stage of the pork tapeworm Taenia solium) is the most common helminthic infection of the nervous system in humans. T. solium is endemic in most developing countries and cysticercosis cases are also seen in developed countries due to immigration and travel. ${ }^{1}$ The life cycle of Taenia solium life involves an intermediate host harboring the parasitic larvae in its tissues, and a definitive host carrying the adult tapeworm in its intestines. Pigs are the most common intermediate host and humans as the sole definitive host. ${ }^{2,3}$ The adult parasite lives in the human small intestine attached to the mucose by its head or scolex, and sheds gravid proglottids containing numerous eggs, which are released through the feces; when the intermediate host ingests these eggs, they hatch in the pig's intestine releasing the embryos or oncospheres that actively cross the intestinal wall to reach a blood vessel and are disseminated by the blood stream to most tissues, where they develop into encysted cysticerci. ${ }^{4,5}$ The life cycle is completed when undercooked pork meat is eaten by a human, and the cysticercus evaginates and attaches to the intestinal wall to develop into an adult tapeworm. ${ }^{6}$

Neurological symptoms due to neurocysticercosis, infection in the central nervous system, are the most common clinical presentation. This is likely due to more clinical 
expression of cysts in the CNS, in comparison to infection in other organs where the cyst goes undetected. ${ }^{7-9}$ It should be noted that even in the CNS, a large proportion of infections will go asymptomatic or oligosymptomatic and thus left undiagnosed. The pre-patent period between infection and symptoms in NCC was estimated between 3 and 5 years by Dixon et al in $1961 .^{10}$ The clinical manifestations of neurocysticercosis are extremely variable and depend on the number, location, size, and evolutive stage of the infecting parasitic larvae, as well as the immune/inflammatory response of the host (1). Of particular importance, parasite location inside the brain parenchyma (intraparenchymal NCC) is less aggressive and is associated with headaches, seizures and epilepsy, while infections locating in the ventricles or subarachnoid spaces (extraparenchymal NCC) are associated with intracranial hypertension and constitute a major driver of mortality and morbidity. ${ }^{11-19}$ Parenchymal NCC accounts for one-third of all epilepsy cases on endemic regions, therefore representing the most important cause of acquired epilepsy worldwide. ${ }^{20}$ Evolution of parenchymal cysts in the CNS goes from a viable, quiescent state that involves little or no inflammation, followed by an immune response of the host with local inflammation, cyst degeneration and then complete resolution or calcification. As mentioned, involution of the cyst is related to inflammation and this effect can cause an exacerbation of neurological symptoms. ${ }^{21}$ Extraparenchymal neurocysticercosis may cause mass effect and hydrocephalus due to blockage (ventricular cysts) or direct compression of the structures related to CSF transit. ${ }^{22-25}$

\section{Diagnostic Difficulties}

Even though neurocysticercosis has a recognized etiologic agent, a definitive histological diagnosis is rarely obtained due to the invasiveness of surgery and poor accessibility of the central nervous system. ${ }^{26}$ Symptoms and signs are extremely variable and do not configure the diagnosis, although both late-onset epilepsy and intracranial hypertension in Taenia solium endemic geographic regions are highly suggestive. Adding to the complexity of the diagnosis, the clinical presentation of NCC may vary according to geographic region, with a single brain enhancing lesion being much more frequent in the Indian subcontinent, associated with a more benign prognosis. Clinical examination, hematological and biochemical laboratories are rarely contributory. As a result, the diagnosis of NCC relies on neuroimaging and immunodiagnostic tests. None of these is absolute and their results are seldom pathognomonic.

\section{Neuroimaging}

Neuroimaging in NCC provides data regarding number, size, localization, perilesional inflammation, and stage of lesions, as well as information on collateral alterations such as hydrocephalus or other signs. ${ }^{27-30}$ Introduced in the 1970s, CT was a major advance in the diagnosis of NCC, allowing the visualization of intracranial structures including NCC lesions, and was followed by the advent of MRI. MRI provides much better image definition as well as the capacity to assess the view in different spatial planes (classically axial, sagittal, and coronal) and solves issues related to the gross hyper density of bone structures on CT due to the strong signal of calcium, that difficult the diagnosis of small lesions that are close to the skull or in the posterior fossa. ${ }^{27,28}$ However, the same strong signal for calcium on $\mathrm{CT}$ allows a much higher sensitivity to observe brain calcifications (Figure 1).

\section{Immunodiagnosis}

Antibody detection using the enzyme-linked immunoelectrotransfer blot assay (EITB, Western blot) using lentil lectin purified parasite glycoprotein antigen (LLGP) is the tool of choice for serological diagnosis of cysticercosis. ${ }^{31}$ Antibodies can be detected on EITB as soon as 5 weeks after infection. ${ }^{32}$ The LLGP-EITB has a sensitivity of $98 \%$ in patients with more than one brain cyst, and a specificity of $100 \%,{ }^{33,34}$ although its sensitivity drops in cases with a single cyst. As much as it is known, the sensitivity of EITB is not affected by HIV infection or immunosuppressive therapies. Antigen detection using a monoclonal antibody-based ELISA can confirm the presence of living parasites (it gives negative results in calcified disease). Its sensitivity is lower and it is frequently negative in patients with one or a few living parasites. It may be useful for therapeutic decisions and to monitor the effect of antiparasitic treatment. ${ }^{35}$

\section{Charts of Diagnostic Criteria for Cysticercosis and Neurocysticercosis}

While clinical descriptions of NCC can be found in the medical literature since the end of the XIX century, the poor diagnostic accuracy of early imaging and serological exams before the availability of CT scan and the LLGPEITB hampered the development of a standard diagnostic approach. The challenge is even bigger if we consider the enormous clinical, immunological and neuroimaging 

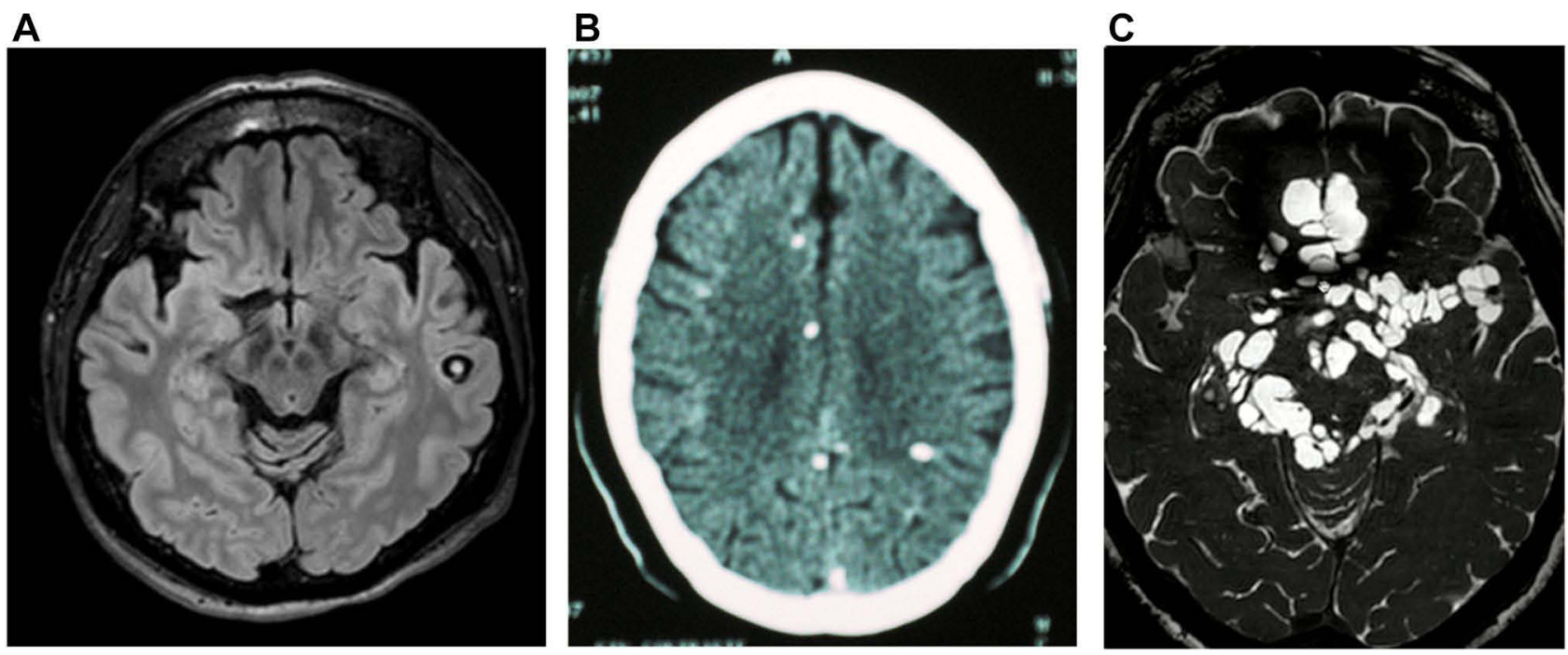

Figure I Parenchymal and extraparenchymal neurocysticercosis. (A) (left): Viable cyst showing its scolex (MRI, FLAIR protocol), (B) (center): calcified cysts (noncontrasted CT scan), and (C) (right): basal subarachnoid cysticercosis (MRI, BFFE protocol).

variability arising from varying numbers, size, localizations, and type of lesions in the central nervous system.

In 1996, a group of experts associated clinical manifestations, imaging, immunologic test and epidemiologic criteria and conceived the first set of diagnostic criteria for human cysticercosis with the purpose of standardizing the diagnostic approach. ${ }^{36}$ This chart classified potential diagnostic criteria in four categories: absolute, major, minor, and epidemiologic.

Absolute criteria included histology (histologic demonstration of the parasite from biopsy of a subcutaneous nodule or brain lesion), direct visualization of an ocular parasite by funduscopic examination, or cystic lesions showing the scolex on CT or MRI. Major criteria included lesions suggestive of neurocysticercosis on neuroimaging studies, positive immunologic tests for the detection of anticysticercal antibodies, or plain X-ray films showing multiple "cigar-shaped" calcifications in thigh and calf muscles. Minor criteria were the presence of subcutaneous nodules on clinical examination, punctuate calcifications on plain X-ray films, suggestive clinical manifestations, or the disappearance of intracranial lesions after a trial with anticysticercal drugs, and finally Epidemiologic criteria included living in a cysticercosis-endemic area, frequent travel to cysticercosis-endemic areas, or a household contact with $T$. solium taeniasis.

These criteria configured three degrees of diagnostic certainty: definitive, probable and possible. A definitive diagnosis requires the presence of one absolute criterion, two major criteria or one major criterion plus two minor and one epidemiological. A probable diagnosis required one major plus two minor criteria, or one major plus one minor plus one epidemiologic, or three minor plus one epidemiologic criteria, whereas a possible diagnosis could be established with one major criterion, two minor criteria or one minor plus one epidemiologic criteria.

The major objective of this initial chart of diagnostic criteria for cysticercosis was to avoid the overdiagnosis of neurocysticercosis in endemic regions, minimizing diagnoses based on clinical manifestations only.

Another concept brought up in the initial 1996 version was that neurocysticercosis is a frequent finding in endemic regions; in these settings asymptomatic NCC can coexist with other neurologic conditions without being the cause of disease. Therefore, finding an image compatible with NCC does not exempt clinicians from the need of ruling out other conditions considered in the differential diagnosis. ${ }^{37-41}$ Also, it brought up the idea that neurocysticercosis should be considered in the differential diagnosis of neurological conditions in non-endemic areas due to increased migration and travel.

2001 Revision. Five years after the initial publication, a larger group of experts convened to reorganize the chart of criteria and propose a new, updated version. After 4 years of experience using the first chart of diagnostic criteria, these were optimized prioritizing signs related to neurologic compromise. ${ }^{42}$ There were no changes in the previously defined absolute criteria, but there were several modifications in the major criteria category. It now included only lesions highly suggestive of neurocysticercosis on neuroimaging 
studies (leaving other compatible lesions aside as a minor criterion), restricted the major immunodiagnosis criterion to cases with a positive EITB only, resolution of intracranial cystic lesions after therapy with albendazole or praziquantel (previously a minor criterion), and spontaneous resolution of small single enhancing lesions. Minor criteria were other lesions compatible with neurocysticercosis on neuroimaging, suggestive clinical manifestations, a positive CSF ELISA for detection of anticysticercal antibodies or cysticercal antigen, or evidence for cysticercosis outside the CNS (replacing the previous criterion of presence of subcutaneous nodules on clinical examination or plain X-ray films showing multiple "cigar-shaped" calcifications in thigh and calf muscles). The epidemiologic criteria remained without changes. In the 2001 version, a definitive diagnosis required one absolute criterion, or two major criteria plus one minor and one epidemiologic criterion; a probable diagnosis was defined by one major plus two minor criteria, or one major plus one minor plus one epidemiologic criteria, or three minor criteria plus one epidemiologic criterion. The lack of reliability of a "possible" degree of certainty led to its exclusion from this version.

This paper was widely cited, with a mean of 49 citations per year from 2002 to 2020 . When evaluated by an external group, the sensitivity and specificity of this version were determined to be of $93.6 \%$ and $81.1 \%$, respectively. Its sensitivity for intraventricular NCC is estimated in $72.7 \%{ }^{43}$

2017 Revision. More than 15 years after the first revision, in 2017 a new version of the diagnosis criteria chart was presented. ${ }^{44}$ The concepts behind this new version were to provide simpler and more operational definitions, and focus on the demonstration of CNS lesions by neuroimaging as the more important diagnostic driver. Thus, criteria were allocated into two groups: those involving imaging, versus those who have clinical manifestations, immunodiagnostic assay confirmation, or epidemiologic context. Independent of this, absolute criteria remained based on direct visualization of the parasite or the histological demonstration (Table 1).

Neuroimaging criteria are now specified and divided in major (cystic lesions without a scolex, enhancing lesions, multilobulated subarachnoid lesions, or typical brain calcifications), confirmative (cyst resolution after antiparasitic treatment, spontaneous resolution of a single enhancing lesion, or migration of a ventricular cyst), or minor (obstructive hydrocephalus or leptomeningeal enhancement). Clinical, immunological or epidemiological criteria were left unchanged except for the unification of welldefined immunodiagnostic tests in a single major criterion. A definitive diagnosis requires one absolute criterion, two major neuroimaging criteria plus any clinical/exposure criteria, one major and one confirmative neuroimaging criteria plus any clinical/exposure criteria, or one major neuroimaging criterion plus two clinical/exposure criteria with the exclusion of other pathologies producing similar neuroimaging findings (note that at least one major neuroimaging criterion is always required). A probable diagnosis is established with one major neuroimaging criterion plus any two clinical/exposure criteria; or one minor neuroimaging criterion plus at least one major clinical/exposure criterion. This latter version is more intuitive and easier to apply in different scenarios and environments, but also urges the need of having clinicians trained in NCC diagnosis, particularly in the differential neuroimaging diagnosis.

\section{Limitations and Potential for Improvement}

Even the more characteristic neuroimaging findings could be mimicked by other conditions, and in many cases, images are not conclusive. The best performing immunodiagnostic tests are poorly available worldwide, and each technique has its own drawbacks. Antibody detection is more sensitive for diagnosis, but cannot distinguish active infections, while antigen detection is limited by the small amounts of circulating antigen produced by the parasites, reducing its sensitivity. Simpler, more reproducible tests based on synthetic antigens or molecular diagnosis are needed to homogenize immunodiagnosis across countries.

Beyond the limited availability of diagnostic tools, other factors contribute to the challenge of making a proper and early diagnosis of neurocysticercosis. In endemic regions, patients may have limited access to medical care, and do not always have access to sophisticated diagnostic methods. In addition, the extreme variability in presenting symptoms and the long pre-patent period can confuse attending clinicians that are not familiar with NCC.

Other authors have criticized the Del Brutto diagnostic criteria. Garg et al $^{45,46}$ noted a series of difficulties when applying the 2001 criteria in the context of the Indian subcontinent, where the vast majority of NCC cases present with a single enhancing lesion. Many other infectious (tuberculoma, mycosis, other parasites) 
Table I Diagnostic Criteria for Human Neurocysticercosis

\begin{tabular}{|c|c|c|}
\hline 2017 & 2001 & 1996 \\
\hline Absolute Criteria & Absolute Criteria & Absolute Criteria \\
\hline $\begin{array}{l}\text { Histological demonstration of the parasite from } \\
\text { biopsy of a brain or spinal cord lesion }\end{array}$ & Similar & $\begin{array}{l}\text { Included subcutaneous nodules, did not } \\
\text { include spinal cord }\end{array}$ \\
\hline Direct visualization of subretinal cysticercus & Similar & SImilar \\
\hline $\begin{array}{l}\text { Conclusive demonstration of a scolex within a cystic } \\
\text { lesion on neuroimaging studies }\end{array}$ & Similar & Similar \\
\hline \multicolumn{3}{|l|}{ Neuroimaging Criteria } \\
\hline Major Neuroimaging Criteria & Other Criteria & Other Criteria \\
\hline $\begin{array}{l}\text { - Cystic lesions without a discernible scolex } \\
\text { - Enhancing lesions } \\
\text { - Multilobulated cystic lesions in the subarachnoid } \\
\text { spaces } \\
\text { - Typical parenchymal brain calcifications }\end{array}$ & $\begin{array}{l}\text { Lesions highly suggestive of } \\
\text { Neurocysticercosis on neuroimaging } \\
\text { studies (major) }\end{array}$ & Similar (major) \\
\hline \multicolumn{3}{|l|}{ Confirmatory Neuroimaging Criteria } \\
\hline $\begin{array}{l}\text { Resolution of cystic lesions after cysticidal drug } \\
\text { therapy }\end{array}$ & Similar (major) & Similar (minor) \\
\hline $\begin{array}{l}\text { Spontaneous resolution of single small enhanced } \\
\text { lesions }\end{array}$ & Similar (major) & Not included \\
\hline $\begin{array}{l}\text { Migration of ventricular cysts documented on } \\
\text { sequential neuroimaging studies }\end{array}$ & Not included & Not included \\
\hline \multicolumn{3}{|l|}{ Minor Neuroimaging Criteria } \\
\hline $\begin{array}{l}\text { Obstructive hydrocephalus (symmetric or } \\
\text { asymmetric) or abnormal enhancement of basal } \\
\text { leptomeninges }\end{array}$ & $\begin{array}{l}\text { Lesions compatible with } \\
\text { Neurocysticercosis on neuroimaging } \\
\text { studies (minor) }\end{array}$ & Not included \\
\hline \multicolumn{3}{|l|}{ Clinical/Exposure Criteria } \\
\hline \multicolumn{3}{|l|}{ Major Clinical/Exposure Criteria } \\
\hline $\begin{array}{l}\text { Detection of specific anti cysticercal antibodies or } \\
\text { cysticercal antigens by well-standardized } \\
\text { immunodiagnostic tests }\end{array}$ & $\begin{array}{l}\text { - Positive serum EITB for the detection of } \\
\text { anti cysticercal antibodies (major) } \\
\text { - Positive CSF ELISA for detection of anti } \\
\text { cysticercal antibodies or cysticercal antigen } \\
\text { (minor) }\end{array}$ & $\begin{array}{l}\text { Positive immunologic tests for the detection } \\
\text { of anti cysticercal antibodies (major) }\end{array}$ \\
\hline Cysticercosis outside the central nervous system & Similar (minor) & $\begin{array}{l}\text { - Plain X-ray films showing multiple "cigar- } \\
\text { shaped" calcifications in thigh and calf } \\
\text { muscles (major) } \\
\text { - Presence of subcutaneous nodules } \\
\text { (without histological confirmation) (minor) } \\
\text { - Evidence of punctuate soft-tissue or } \\
\text { intracranial calcification on plain X-ray films } \\
\text { (minor) }\end{array}$ \\
\hline $\begin{array}{l}\text { Evidence of a household contact with } T \text {. solium } \\
\text { infection }\end{array}$ & Similar (epidemiologic) & Similar (epidemiologic) \\
\hline
\end{tabular}


Table I (Continued).

\begin{tabular}{|c|c|c|}
\hline 2017 & 2001 & 1996 \\
\hline \multicolumn{3}{|l|}{ Minor Clinical/Exposure Criteria } \\
\hline $\begin{array}{l}\text { Clinical manifestations suggestive of } \\
\text { neurocysticercosis }\end{array}$ & Similar (minor) & Similar (minor) \\
\hline $\begin{array}{l}\text { Individuals coming from or living in an area where } \\
\text { cysticercosis is endemic }\end{array}$ & Similar (epidemiologic) & Similar (epidemiologic) \\
\hline Not included & $\begin{array}{l}\text { History of frequent travel to disease } \\
\text { endemic areas (epidemiologic) }\end{array}$ & $\begin{array}{l}\text { History of frequent travel to cysticercosis- } \\
\text { endemic areas (epidemiologic) }\end{array}$ \\
\hline
\end{tabular}

Note: Adapted from Journal of the Neurological Sciences, 372, O.H. Del Brutto, T.E. Nash, A.C. White, V. Rajshekhar, P.P.Wilkins, G. Singh, C.M. Vasquez, P. Salgado,R.H. Gilman, H.H.Garcia, Revised diagnostic criteria for neurocysticercosis, 202-210, Copyright 2017, with permission from Elsevier. ${ }^{44}$

and neoplastic etiologies can produce similar findings on neuroimaging, and the performance of EITB in this type of NCC is lower. They also recommended to earmark some situations where NCC is less likely such as middle or old age, pre-existing HIV, tuberculosis or malignancy, or evidently abnormal neurological examination. Others find the chart difficult to apply and argue that it will be poorly sensitive for extraparenchymal $\mathrm{NCC}^{41}$ In addition, Gabriel et al suggest to add serum antigen detection as a major criterion in resource poor countries, where neuroimaging is not available. ${ }^{47}$ While the idea of multiple charts to adapt to individual scenarios does not align with the purpose of having a unified set of diagnostic criteria, the other arguments have been discussed ${ }^{48}$ or approached in the 2017 revision.

\section{Impact}

In the past 25 years, the introduction of standard diagnostic criteria for cysticercosis have contributed to homogenize case definition, allowing sound comparisons of case series and published literature. The 2001 version is the most quoted paper on NCC, with 975 citations, and is used by most medical textbooks. In endemic countries, where clinicians are more familiar with the disease, and in developed countries, where cases present due to travel and migration, the availability of a structured diagnosis allows better case confirmation and systematization.

\section{Disclosure}

H.G. contributed to the 2001 and the 2017 versions of the diagnostic criteria for neurocysticercosis revised in this manuscript. The authors report no other conflicts of interest in this work.

\section{References}

1. Garcia HH, Gonzalez AE, Gilman RH. Taenia solium cysticercosis and its impact in neurological disease. Clin Microbiol Rev. 2020;33: e00085-e00119.

2. Flisser A. State of the art of Taenia solium as compared to Taenia asiatica. Korean J Parasitol. 2013;51:43-49.

3. Flisser A. Taeniasis and cysticercosis due to Taenia solium. Progr Clin Parasitol. 1994;4:77-116.

4. Mendlovic F, Garza-Rodríguez A, Carrillo-Farga J, et al. From stillness to motion: 80 years after the first description of Taenia solium oncosphere hatching. Parasit Vectors. 2014;7:12.

5. White AC, Baig S, Robinson P. Taenia saginata oncosphere excretory/secretory peptidases. J Parasitol. 1996;82:7-10.

6. Kean BH, Mott KE, Russell AJ Tropical Medicine and Parasitology. Classic investigations. Ithaca, NY: Cornell University Press; 1978.

7. Gonzalez AE, Gauci CG, Barber D, et al. Vaccination of pigs to control human neurocysticercosis. Am J Trop Med Hyg. 2005;72:837-839.

8. Brailsford JF. Cysticercus cellulosae, its radiographic detection in the musculature and the central nervous system. $\operatorname{Br} J$ Radiol. 1941;14:79-93.

9. Morrison WK. Cysticercosis in twin brothers aged 13 years. Br Med J. 1934;1:13-14.

10. Dixon HBF, Lipscomb FM Cysticercosis: an analysis and follow-up of 450 cases. Special Report Series, Medical Research Council; 1961:299:1-58.

11. Cho TA. Helminthic Infections of the central nervous system. Continuum (Minneapolis, Minn). 2018;24:1489-1511.

12. Elliott EJ, Landaker EJ. Worsening migraine due to neurocysticercosis. Cleve Clin J Med. 2017;84:196-198.

13. Fogang YF, Camara M, Diop AG, Ndiaye MM. Cerebral neurocysticercosis mimicking or comorbid with episodic migraine? $B M C$ Neurol. 2014;1(14):138.

14. Garcia HH, Del Brutto OH; Cysticercosis Working Group in Peru. Antiparasitic treatment of neurocysticercosis - The effect of cyst destruction in seizure evolution. Epilepsy Behav. 2017;76:158-162.

15. Garcia HH, Nash TE, Del Brutto OH. Clinical symptoms, diagnosis, and treatment of neurocysticercosis. Lancet Neurol. 2014;13:1202-1215. 
16. Millogo A, Kongnyu Njamnshi A, Kabwa-Pierre Luabeya M. Neurocysticercosis and epilepsy in sub-Saharan Africa. Brain Res Bull. 2019;145:30-38.

17. Nash TE, Mahanty S, Loeb JA, et al. Neurocysticercosis: a natural human model of epileptogenesis. Epilepsia. 2015;56:177-183.

18. Saito EK, Mehta B, Wang F, Nakamoto B, McMurtray AM. headaches more common among epilepsy sufferers with neurocysticercosis than other structural brain lesions. Hawaii J Med Public Health. 2017;76:152-155.

19. Ghasemi R, Rowe A, Shah R, Venkatesan P, England TJ. Neurocysticercosis presenting as a 'Stroke Mimic'. Acute Med. 2016;15:79-83.

20. Medina MT, Duron RM, Martínez L, et al. Prevalence, incidence, and etiology of epilepsies in rural Honduras: the Salamá Study. Epilepsia. 2005;46:124-131.

21. Nash TE, Mahanty S, Garcia HH; Cysticercosis Group in Peru. Corticosteroid use in neurocysticercosis. Expert Rev Neurother. 2011;11:1175-1183.

22. Ghosh S, Al-Khalili R, Liu JK, Slasky SE. Paradoxical migrating cyst: an unusual presentation of intraventricular neurocysticercosis with a coincidental pituitary adenoma. $J$ Clin Neurosci. 2014;21:1066-1068.

23. Rana S, Prasad A, Brar R, Rathore DS, Dwivedi A. Caught in the act: migrating intraventricular neurocysticercosis causing intermittent unilateral hydrocephalus due to foramen of Monro obstruction. Acta Neurol Belg. 2017;118:509-511.

24. Shah A, Vutha R, Sankhe S, Goel A. Transventricular migration of neurocysticercosis. World Neurosurg. 2017;105:1043.e11-1043.e13.

25. Bustos JA, Garcia HH, Del Brutto OH. reliability of diagnostic criteria for neurocysticercosis for patients with ventricular cystic lesions or granulomas: a systematic review. Am J Trop Med Hyg. 2017;97:653-657.

26. Del Brutto $\mathrm{OH}$. Diagnostic criteria for neurocysticercosis, revisited. Pathog Glob Health. 2012;106:299-304.

27. Dumas JL, Visy JM, Belin C, et al. Dumas, M. Parenchymal neurocysticercosis: follow-up and staging by MRI. Neuroradiology. 1997;39:12-18.

28. Garcia $\mathrm{HH}$, Del Brutto $\mathrm{OH}$. Imaging findings in neurocysticercosis. Acta Trop. 2003;87:71-78.

29. Hernández RD, Durán BB, Lujambio PS. Magnetic resonance imaging in neurocysticercosis. Top Magn Reson Imaging. 2014;23:191-198.

30. Lerner A, Shiroishi MS, Zee CS, Law M, Go JL. Imaging of neurocysticercosis. Neuroimaging Clin N Am. 2012;22:659-676.

31. Rodriguez S, Wilkins P, Dorny P. Immunological and molecular diagnosis of cysticercosis. Pathog Glob Health. 2012;106:286-298.

32. Tsang VC, Pilcher JA, Zhou W, et al. Efficacy of the immunoblot assay for cysticercosis in pigs and modulated expression of distinct $\mathrm{IgM} / \mathrm{IgG}$ activities to Taenia solium antigens in experimental infections. Vet Immunol Immunopathol. 1991;29:69-78.
33. Arroyo G, Rodriguez S, Lescano AG, et al. Antibody banding patterns of the enzyme-linked immunoelectrotransfer blot and brain imaging findings in patients with neurocysticercosis. Clin Inf Dis. 2018;66:282-288.

34. Garcia HH, O’Neal SE, Noh J, Handali S; Cysticercosis Working Group in Peru. Laboratory diagnosis of neurocysticercosis (Taenia solium). J Clin Microbiol. 2018;56:e00424-18.

35. Zea-Vera A, Cordova EG, Rodriguez S, et al. Parasite antigen in serum predicts the presence of viable brain parasites in patients with apparently calcified cysticercosis only. Clin Inf Dis. 2013;57:e154e159.

36. Del Brutto OH, Wadia NH, Dumas M, Cruz M, Tsang VCW, Schantz PM. Proposal of diagnostic criteria for human cysticercosis and neurocysticercosis. J Neurol Sci. 1996;142:1-6.

37. Del Brutto OH. Single parenchymal brain cysticercus in the acute encephalitic phase: definition of a distinct form of neurocysticercosis with benign prognosis. $J$ Neurol Neurosurg Psychiatry. 1995;58:247-249.

38. Del Brutto OH. Late-onset epilepsy in Ecuador: aetiology and clinical features in 225 patients. J Trop Geogr Neurol. 1991;1:31-34.

39. Del Brutto OH. Cysticercosis mimicking brain tumor: the role of albendazole as a diagnostic tool. Clin Neurol Neurosurg. 1995;97:256-258.

40. Del Brutto OH, Santibañez R, Noboa CA, et al. Epilepsy due to neurocysticercosis: analysis of 203 patients. Neurology. 1992;42:389-392.

41. Del Brutto OH, Sotelo J. Neurocysticercosis: an update. Rev Infect Dis. 1988;10:1075-1087.

42. Del Brutto OH, Rajshekhar V, White AC Jr, et al. Proposed diagnostic criteria for neurocysticercosis. Neurology. 2001;57:177-183.

43. Carpio A, Fleury A, Romo ML, et al. New diagnostic criteria for neurocysticercosis: reliability and validity. Ann Neurol. 2016;80:434-442.

44. Del Brutto OH, Nash TE, White AC, et al. Revised diagnostic criteria for neurocysticercosis. J Neurol Sci. 2017;372:202-210.

45. Garg RK. Proposed diagnostic criteria for neurocysticercosis. Neurology. 2002;58:1315-1316.

46. Garg RK. Diagnostic criteria for neurocysticercosis: some modifications are needed for Indian patients. Neurol India. 2004;52:171-177.

47. Gabriel S, Blocher J, Dorny P, et al. Added value of antigen ELISA in the diagnosis of neurocysticercosis in resource poor settings. PLoS Negl Trop Dis. 2012;6:e1851.

48. Gilman RH. Diagnostic criteria for neurocysticercosis - a difficult update. Nat Rev Neurol. 2016;12:560-561.

\section{Publish your work in this journal}

Research and Reports in Tropical Medicine is an international, peerreviewed, open access journal publishing original research, case reports, editorials, reviews and commentaries on all areas of tropical medicine, including: Diseases and medicine in tropical regions; Entomology; Epidemiology; Health economics issues; Infectious disease; Laboratory science and new technology in tropical medicine;
Parasitology; Public health medicine/health care policy in tropical regions; and Microbiology. The manuscript management system is completely online and includes a very quick and fair peer-review system. Visit http://www.dovepress.com/testimonials.php to read real quotes from published authors. 